\title{
BONFERRONI-TYPE INEQUALITIES
}

\author{
ELAINE RECSEI* AND \\ E. SENETA, University of Sydney
}

\begin{abstract}
We derive the Sobel-Uppuluri and Galambos-type extensions of the Bonferroni bounds, and further extensions of the same nature, as consequences of a single non-probabilistic inequality. The methodology follows that of Galambos.
\end{abstract}

JORDAN INEQUALITIES; GALAMBOS INEQUALITIES; INVERSION FORMULA; BINOMIAL MOMENTS

\section{Unified treatment and extensions}

Let $w_{t}, t=0,1, \cdots, n$ be non-negative numbers. Define

$$
A_{k, n}=\sum_{t=k}^{n}\left(\begin{array}{l}
t \\
k
\end{array}\right) w_{t}, \quad k \geqq 0 \quad\left(A_{k, n}=0, k>n\right) .
$$

Then, following Galambos [3], pp. 18-20, [2], p. 580,

$$
\sum_{k=0}^{a}(-1)^{k}\left(\begin{array}{c}
k+r \\
r
\end{array}\right) A_{k+r, n}=w_{r}+(-1)^{a} \sum_{s=r+a+1}^{n}\left(\begin{array}{c}
s-r-1 \\
a
\end{array}\right)\left(\begin{array}{l}
s \\
r
\end{array}\right) w_{s}
$$

for $0 \leqq a \leqq n-r-1$, and

$$
\sum_{s=r+a+1}^{n}\left(\begin{array}{c}
s-r-1 \\
a
\end{array}\right)\left(\begin{array}{l}
s \\
r
\end{array}\right) w_{s} \geqq \frac{a+1}{n-r}\left(\begin{array}{c}
a+1+r \\
r
\end{array}\right) A_{a+1+r, n} .
$$

Using (3) in (2) yields the bounds for $0 \leqq r \leqq n, u \geqq 0,\left(\sum_{s=r}^{r-1}=0\right)$ :

$$
\begin{aligned}
& \sum_{s=r}^{r+2 u-1}(-1)^{s-r}\left(\begin{array}{l}
s \\
r
\end{array}\right) A_{s, n}+\frac{2 u}{n-r}\left(\begin{array}{c}
r+2 u \\
r
\end{array}\right) A_{r+2 u, n} \leqq w_{r} \\
& \leqq \sum_{s=r}^{r+2 u}(-1)^{s-r}\left(\begin{array}{l}
s \\
r
\end{array}\right) A_{s, n}-\frac{2 u+1}{n-r}\left(\begin{array}{c}
r+2 u+1 \\
r
\end{array}\right) A_{r+2 u+1, n}
\end{aligned}
$$

keeping in mind (for $r+2 u \geqq n$ ) the inversion of the relation (1):

$$
w_{r}=\sum_{s=r}^{n}(-1)^{s-r}\left(\begin{array}{l}
s \\
r
\end{array}\right) A_{s, n}, \quad 0 \leqq r \leqq n .
$$

Received 21 January 1987.

* Postal address: Department of Mathematical Statistics, University of Sydney, NSW 2006 Australia. 
Now let $y_{t}, t=1, \cdots, n$, be arbitrary non-negative numbers, and define $S_{k, n}$ by

$$
S_{k, n}=\sum_{t=k}^{n}\left(\begin{array}{l}
t-1 \\
k-1
\end{array}\right) y_{t}, \quad k \geqq 1 \quad\left(S_{k, n}=0, k>n\right) .
$$

Since $\left(\begin{array}{c}t-1 \\ k-1\end{array}\right)=(k / t)\left(\begin{array}{l}t \\ k\end{array}\right)$, it follows that

$$
S_{k, n} / k=\sum_{t=k}^{n}\left(\begin{array}{l}
t \\
k
\end{array}\right)\left(y_{t} / t\right), \quad k \geqq 1 .
$$

Thus putting $w_{0}=0$ and taking in (1) and (4) for $k \geqq 1, A_{k, n}=S_{k, n} / k$, and $w_{t}=y_{t} / t$, $1 \leqq t \leqq n$, it follows that for $1 \leqq r \leqq n, u \geqq 0$,

$$
\begin{gathered}
\sum_{s=r}^{r+2 u-1}(-1)^{s-r}\left(\begin{array}{c}
s-1 \\
r-1
\end{array}\right) S_{s, n}+\frac{2 u}{n-r}\left(\begin{array}{c}
r+2 u-1 \\
r-1
\end{array}\right) S_{r+2 u, n} \leqq y_{r} \\
\leqq \sum_{s=r}^{r+2 u}(-1)^{s-r}\left(\begin{array}{c}
s-1 \\
r-1
\end{array}\right) S_{s, n}-\frac{2 u+1}{n-r}\left(\begin{array}{c}
r+2 u \\
r-1
\end{array}\right) S_{r+2 u+1, n}
\end{gathered}
$$

again keeping in mind (5).

Turning now to a probabilistic setting, let $A_{1}, \cdots, A_{n}$ be a sequence of events on a probability space, let $B_{r, n}, 0 \leqq r \leqq n$, be the event that exactly $r$ of the $A$ 's occur, and let $P_{[r]}=P\left(B_{r, n}\right)$. Let $S_{k, n}=\sum P\left(A_{i_{1}} A_{i_{2}} \cdots A_{i_{k}}\right)$ where the sum is over all subscripts satisfying $1 \leqq i_{1}<i_{2}<\cdots<i_{k} \leqq n$. Then it is well known (defining $S_{0, n}$ as 1 ) that:

$$
S_{k, n}=\sum_{t=k}^{n}\left(\begin{array}{l}
t \\
k
\end{array}\right) P_{[t]}, \quad k \geqq 0,
$$

so putting $w_{t}=P_{[t]}$ and $A_{k, n}=S_{k, n}$ in (1), (4) yields the Sobel-Uppuluri-Galambos inequalities ([3], p. 20; [5]), Further, it is well known that if we put

$$
P_{(r)}=\sum_{s=r}^{n} P_{[s]}
$$

then for $1 \leqq r \leqq n$,

$$
S_{k, n}=\sum_{t=k}^{n}\left(\begin{array}{l}
t-1 \\
k-1
\end{array}\right) P_{(t)}, \quad k \geqq 1
$$

so putting $y_{t}=P_{(t)}$ in (6) and (7) yields the Galambos bounds ([2], [6]) for $P_{(r)}, r \geqq 1$. The direction of generalisation is now clear. For example, for $n \geqq t \geqq 2$, put

$$
P_{\{t\}}=\sum_{s=t}^{n} P_{(s)}=\left(\sum_{h=r}^{n}(h-r+1) P_{[h]}\right) \text {. }
$$

Substituting for $P_{(s)}$ from the inversion formula (5), i.e.

$$
P_{(s)}=\sum_{k=s}^{n}(-1)^{k-s}\left(\begin{array}{l}
k-1 \\
k-s
\end{array}\right) S_{k, n}, \quad 1 \leqq s \leqq n,
$$


and using a combinatorial identity we obtain

$$
P_{\{t\}}=\sum_{k=t}^{n}(-1)^{k-t}\left(\begin{array}{l}
k-2 \\
k-t
\end{array}\right) S_{k, n},
$$

i.e.

$$
P_{\{t\}} /\left(t(t-1)=\sum_{k=t}^{n}(-1)^{k-t}\left(\begin{array}{l}
k \\
t
\end{array}\right) S_{k, n} / k(k-1),\right.
$$

whence by the inversion formula to (5), viz. (1),

$$
S_{k, n}=\sum_{r=k}^{n}\left(\begin{array}{l}
r-2 \\
k-2
\end{array}\right) P_{\{r\}}, k \geqq 2 .
$$

Hence for $2 \leqq r \leqq n, u \geqq 0$,

$$
\begin{gathered}
\sum_{s=r}^{r+2 u-1}(-1)^{s-r}\left(\begin{array}{c}
s-2 \\
r-2
\end{array}\right) S_{s, n}+\frac{2 u}{n-r}\left(\begin{array}{c}
r+2 u-2 \\
r-2
\end{array}\right) S_{r+2 u, n} \leqq P_{\{r\}} \\
\leqq \sum_{s=r}^{r+2 u}(-1)^{s-r}\left(\begin{array}{l}
s-2 \\
r-2
\end{array}\right) S_{s, n}-\frac{2 u+1}{n-r}\left(\begin{array}{c}
r+2 u \\
r-1
\end{array}\right) S_{r+2 u+1, n} .
\end{gathered}
$$

\section{The method of polynomials}

In the general vein of Galambos' conceptualization of the Bonferroni inequalities ([1]), the following deductions can be made from extending slightly the argument in [4]. Suppose for all $p, 0 \leqq p \leqq 1$ and all integers $m \geqq 0$,

$$
(1-p)^{m} \leqq \sum_{k=0}^{m} c_{k}(m)\left(\begin{array}{l}
m \\
k
\end{array}\right) p^{k} \quad\left(\text { where } c_{0}(0)=1\right) .
$$

Then with the notation of the above probabilistic setting

$$
P_{[r]}=P\left(B_{r, n}\right) \leqq \sum_{z=r}^{n} c_{z-r}(n-r)\left(\begin{array}{l}
z \\
r
\end{array}\right) S_{z, n} .
$$

(If the inequality in the supposition (8) is reversed, it is reversed in (9).)

Thus Theorem 4 in [4] can be written down directly from its Lemma 2. Thus, once the inequalities (in the form of Taylor expansion with remainder):

$$
\begin{aligned}
& \sum_{k=0}^{2 u-1}\left(\begin{array}{c}
m \\
k
\end{array}\right)(-p)^{k}+\left(\begin{array}{c}
m \\
2 u
\end{array}\right)(-p)^{2 u} \leqq(1-p)^{m} \\
\leqq & \sum_{k=0}^{2 u}\left(\begin{array}{c}
m \\
k
\end{array}\right)(-p)^{k}+\frac{2 u+1}{m}\left(\begin{array}{c}
m \\
2 u+1
\end{array}\right)(-p)^{2 u+1}
\end{aligned}
$$

have been obtained for $m \geqq 0,0 \leqq p \leqq 1$, the Sobel-Uppuluri-type bounds for $P_{[r]}$ follow immediately.

\section{An application}

We give in Table 1 below some data on performance of first-year students in the Faculty of Science at the University of Sydney in the 1980 annual examinations. The intention is to illustrate use of the Sobel-Uppuluri-Galambos bounds (4) in a real situation where the events $A_{1}, \cdots, A_{n}$ are not exchangeable, and effectiveness of prediction of $P_{[0]}$ via partial knowledge is the object. 
TABLE 1

\begin{tabular}{|c|c|c|c|c|}
\hline & \multicolumn{3}{|c|}{ Proportions passing } & \multirow{2}{*}{$\begin{array}{c}\text { Remaining } \\
\text { subject }\end{array}$} \\
\hline & Mathematics & Physics & Chemistry & \\
\hline Mathematics & $0 \cdot 86$ & & & \\
\hline Physics & $0 \cdot 72$ & $0 \cdot 74$ & & \\
\hline Chemistry & $0 \cdot 65$ & $0 \cdot 61$ & $0 \cdot 67$ & \\
\hline Remaining subject & $0 \cdot 72$ & $0 \cdot 65$ & $0 \cdot 62$ & $0 \cdot 79$ \\
\hline \multicolumn{5}{|l|}{ Proportions passing: } \\
\hline \multicolumn{4}{|c|}{ Mathematics, Physics, Chemistry } & 0.60 \\
\hline \multicolumn{4}{|c|}{ Mathematics, Physics, Remaining subject } & 0.64 \\
\hline \multicolumn{4}{|c|}{ Mathematics, Chemistry, Remaining subject } & $0 \cdot 60$ \\
\hline \multicolumn{4}{|c|}{ Physics, Chemistry, Remaining subject } & 0.57 \\
\hline \multicolumn{4}{|c|}{ Mathematics, Physics, Chemistry, Remaining subject } & $0 \cdot 57$ \\
\hline
\end{tabular}

If we take $A_{1}=$ fail mathematics, $A_{2}=$ fail physics, $A_{3}=$ fail chemistry, $A_{4}=$ fail remaining subject, we wish to bound the probability of passing all subjects, $P_{[0]}=0 \cdot 57$.

If the Sobel-Uppuluri-Galambos bounds are used in conjunction with 'ordinary' Bonferroni bounds 'of the same order' in the manner of [3], p. 27, then the results are:

$\begin{array}{lccc} & u=0 & u=1 & u=2 \\ \text { Lower bound } & 0.06 & \mathbf{0 . 4 6} & 0.50 \\ \text { Upper bound } & \mathbf{0 . 7 7} & \mathbf{0 . 8 5} & \mathbf{0 . 5 9}\end{array}$

where bounds of form (4) with $r=0, n=4$ are shown in bold type. Thus even in the case $u=0$, where only the $P\left(A_{i}\right), i=1, \cdots, 4$ are used, the upper bound is a useful approximation in our setting, although the upper bound $\min _{i} P\left(\bar{A}_{2}\right)=0.67$ is better.

\section{References}

[1] Galambos, J. (1975) Methods for proving Bonferroni-type inequalities. J. London Math. Soc. (2) 9, 561-564.

[2] Galambos, J. (1977) Bonferroni inequalities. Ann. Prob. 5, 577-581. York.

[3] Galambos, J. (1978) The Asymptotic Theory of Extreme Order Statistics. Wiley, New

[4] Galambos, J. AND Mucci, R. (1980) Inequalities for linear combinations of binomial moments. Publ. Math. (Debrecen) 27, 263-268.

[5] SoBel, M. AND UPPULURI, V. R. R. (1972) On Bonferroni-type inequalities of the same degree for the probability of unions and intersections. Ann. Math. Statist. 43, 1549-1558.

[6] WALKER, A. M. (1981) On the classical Bonferroni inequalities and the corresponding Galambos inequalities. J. Appl. Prob 18, 757-763. 